\title{
Immunotherapy for Lung Cancer: Many Questions Yet to Be Answered
}

\author{
David S. Ettinger, MD
}

I have been treating patients with lung cancer for more than 40 years, and since the first publication of the NCCN Clinical Practice Guidelines in Oncology (NCCN Guidelines) for Non-Small Cell Lung Cancer (NSCLC) in 1996, ${ }^{1}$ significant progress has been made in the systemic therapy for advanced NSCLC. However, the latest advance and most hopeful therapy for patients with lung cancer is immunotherapy. With immunotherapy, we can say, "the future is now!"

We can look at a number of milestones that have brought us to this point and will inform the future:

- In October 2015, Vice President Joe Biden announced his Cancer Moonshot initiative, which describes increased funding from both the government and private sector to combat cancer.

- In January 2016, billionaire Patrick Soon-Shiong announced an industry-led moonshot program aimed at testing combinations of cancer immunotherapy drugs in clinical trials.

- In March 2016, Johns Hopkins announced the formation of the BloombergKimmel Institute for Cancer Immunotherapy with a \$125 million investment.

- In April 2016, billionaire Sean Parker, cofounder of Napster and first president of Facebook, announced he's giving \$250 million to form the Parker Institute for Cancer Immunotherapy. This institute will bring together immunologists from 6 cancer centers nationwide, including Memorial Sloan Kettering Cancer Center, Stanford University, The University of Texas MD Anderson Cancer Center, University of California Los Angeles, UCSF Helen Diller Family Comprehensive Cancer Center, and the University of Pennsylvania, to develop immunotherapy to fight cancer.

Of course, there is a need to improve therapy for all cancers, but this is especially true for lung cancer because it is the most common cause of cancer mortality globally. In 2012, there were 1.59 million deaths (19.4\% total) worldwide from lung cancer. ${ }^{2}$ Further, it is the leading cause of cancer death in men and women in the United States, with an estimated death rate of 158,080 (85,920 men; 72,160 women) in $2016 .{ }^{3}$

The use of immune checkpoint inhibitors is a promising immunotherapy approach to treat patients with lung cancer. Although $\mathrm{T}$ cells in the body can recognize and kill cancer cells, the cancer cells can evade immune attack by the $T$ cells by expressing PD-L1, which dampens the antitumor immune response. By blocking PD-1 and PDL1, we can reactivate the immune system to kill the cancer cells. ${ }^{4}$

In March 2015, the FDA approved the PD-1 checkpoint inhibitor nivolumab, which binds to the PD-1 receptor and blocks its interaction with PD-L1 and PD-L2, for the treatment of advanced squamous cell lung cancer that progressed on first-line platinum-based chemotherapy. This approval was based on the results of a phase III trial (CheckMate 017) in which 272 patients were randomized to receive either nivolumab, 3 $\mathrm{mg} / \mathrm{kg}$ intravenously every 2 weeks or docetaxel, $75 \mathrm{mg} / \mathrm{m}^{2}$ intravenously every 3 weeks. ${ }^{5}$ For nivolumab versus docetaxel, the overall response rate (ORR) was $20 \%$ versus $9 \%$, and progression-free survival (PFS) was 3.5 versus 2.8 months, respectively (hazard ratio $[\mathrm{HR}], 0.62 ; P<.001)$. Overall survival (OS) was 9.2 versus 6.0 months (HR, 0.59; $P<.001$ ) and 1 -year survival rates were $42 \%$ versus $24 \%$, respectively.

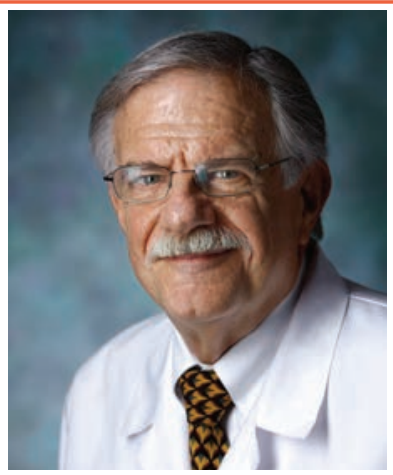

David S. Ettinger, MD

David S. Ettinger, MD, is the Alex Grass Professor of Oncology and Professor of Medicine, Otolaryngology-Head and Neck Surgery, Obstetrics and Gynecology, and Radiation Oncology and Molecular Radiation Sciences at The Johns Hopkins University School of Medicine.

Dr. Ettinger has served as a consultant for dozens of organizations. He is widely published and has authored or co-authored more than 200 articles for journals such as The New England Journal of Medicine, Journal of Clinical Oncology, and Cancer.

Dr. Ettinger is a member of the NCCN Lung Cancer Screening Panel and Chair of the NCCN Non-Small Cell Lung Cancer Panel, Thymic Carcinomas Panel, Occult Primary Cancers Panel, and Antiemesis Panel. $\mathrm{He}$ is a member of the editorial board for JNCCN and serves as a member of several committees and advisory boards for the NCCN Oncology Research Program. Dr. Ettinger also serves as the Chair of the NCCN Guidelines Steering Committee. He also serves on the NCCN International Program Steering Committee, the NCCN Educational Programs Advisory Committee, and the NCCN Board of Directors. 
The most common side effects of nivolumab were fatigue, musculoskeletal pain, decreased appetite, cough, and constipation. Eighty-three patients had tumor samples suitable for PD-L1 expression analysis. Across the prespecified expression levels $(\geq 1 \%$, $\geq 5 \%$, and $\geq 10 \%$ ), PD-L1 expression was not prognostic or predictive of any of the efficacy end points.

The FDA also approved the PD-L1 IHC 28-8 pharmDx test (Dako, Carpinteria, CA) to detect PD-L1 expression levels to help physicians determine which patients may benefit from treatment with nivolumab, but the testing was not mandatory before treatment initiation. Also supporting the FDA approval were the results of a large phase I experience (117 patients) with nivolumab. Although the ORR was $15 \%$ at the time of analysis, 10 of 17 patients who showed a response $(59 \%)$ had response durations of 6 months or longer. At publication, 3-year survival was $18 \%{ }^{6}$

In October 2015, the FDA approved nivolumab to treat nonsquamous cell carcinoma of the lung based on the results of a phase III trial (CheckMate 057) in which patients with nonsquamous carcinoma of the lungs received either nivolumab or docetaxel. ${ }^{7}$ In this study, the ORR was $19.2 \%$ for nivolumab versus $12.4 \%$ for docetaxel, and PFS was 2.3 months for nivolumab versus 4.2 months for docetaxel (HR, $0.92 ; P=.31)$. OS was 12.2 versus $9.4 \%(\mathrm{HR}, 0.73 ; P \leq .0015)$ and 1 -year $O S$ rates were $51 \%$ and $39 \%$, respectively.

Also in October 2015, the FDA approved the PD-1 checkpoint inhibitor pembrolizumab to treat patients with metastatic NSCLC whose tumors expressed the PD-L1 protein in the companion diagnostic. The approval was based on results of a phase I trial (KEYNOTE-001), ${ }^{8}$ in which various doses-2 or $10 \mathrm{mg} / \mathrm{kg}$ every 3 weeks or $10 \mathrm{mg} / \mathrm{kg}$ every 2 weeks - were given across several cohorts of patients who underwent pretreatment or were treatment-naïve. PD-L1 expression was evaluated using immunohistochemistry on fresh tissue specimens, and only patients with membranous staining greater than $1 \%$ were eligible for the study.

The FDA approved pembrolizumab based on a subgroup analysis of 61 patients whose tumor overexpressed PD-L1 on a companion diagnostic test called PDL1 IHC 22C3 pharmDx (Dako). After treatment with pembrolizumab, tumor size decreased in 24 patients (41\%), and the effect lasted up to 9.1 months. In the overall patient population, the ORR, PFS, and OS were 19.4\%, 3.7 months, and 12 months, respectively. The PD-L1 expression rates were grouped according to $1 \%$ or lower, between $1 \%$ and $49 \%$, and $50 \%$ or greater; $23 \%$ of patients had a high ORR and longer PFS and OS. The most common side effects were fatigue, pruritus, and decreased appetite.

A phase II/III study was conducted of pembrolizumab versus docetaxel for PD-L1positive NSCLC after failure of platinum-based chemotherapy (KEYNOTE-010). ${ }^{9}$ This study compared pembrolizumab, $2 \mathrm{mg} / \mathrm{kg}$ intravenously every 3 weeks versus 10 $\mathrm{mg} / \mathrm{kg}$ intravenously every 3 weeks versus docetaxel, $75 \mathrm{mg} / \mathrm{m}^{2}$ intravenously every 3 weeks. The primary end points were PFS and OS, looking at the end points related to PD-L1 expression in the $50 \%$ or greater stratum and the $1 \%$ or greater population.

Among patients with PD-L1 expression of $50 \%$ or greater, OS for pembrolizumab at $2 \mathrm{mg} / \mathrm{kg}$ was 14.9 months (HR, 0.54; $P=.0002)$ versus 17.3 months for permbrolizumab at $10 \mathrm{mg} / \mathrm{kg}(\mathrm{HR}, 0.50 ; \mathrm{P}<.0001)$ and 8.2 months for docetaxel. PFS was significantly longer with pembrolizumab at $2 \mathrm{mg} / \mathrm{kg}$ and pembrolizumab at 10 $\mathrm{mg} / \mathrm{kg}$ than with docetaxel ( 5.0 and 5.2 vs 4.1 months, respectively; HR, $0.59 ; P<.0001$ ). In patients with PD-L1 expression of $1 \%$ or greater, OS for pembrolizumab at $2 \mathrm{mg}$ / $\mathrm{kg}$ was 10.4 months ( $\mathrm{HR}, 0.71 ; P=.0008)$ versus 12.7 months (HR, $0.61 ; P<.0001$ ) for pembrolizumab at $10 \mathrm{mg} / \mathrm{kg}$ and was at 8.5 months for docetaxel. PFS was 3.9 , 4.0 , and 4.0 months, respectively. Comparable efficacy was seen for pembrolizumab, 2 and $10 \mathrm{mg} / \mathrm{kg}$ intravenously every 3 weeks. The data also validated the PD-L1 
expression in advanced NSCLC. The current approved dose of pembrolizumab is 2 $\mathrm{mg} / \mathrm{kg}$ intravenously every 3 weeks.

Currently, only nivolumab and pembrolizumab are approved for the treatment of lung cancer in the second-line setting. However, studies are ongoing evaluating nivolumab in the first-line setting and in combination with other agents. CheckMate 012 is a multi-arm phase I trial evaluating nivolumab versus nivolumab plus ipilumumab in chemotherapy-naïve patients with lung cancer.

Other checkpoint inhibitors are being evaluated in the treatment of patients with lung cancer. Atezolizumab (MPDL3280A) is an antibody targeting PD-L1 rather than PD-1. A phase III study is underway to include the agent with combination chemotherapy in chemotherapy-naïve stage IV nonsquamous NSCLC. Another, similar study includes the addition of bevacizumab; other phase III studies are atezolizumab versus chemotherapy for patients with PD-L1-positive chemotherapynaïve stage IV nonsquamous cell NSCLC and 2 other similar separate studies involving patients with squamous cell NSCLC; one study includes PD-L1-positive disease.

Another checkpoint inhibitor, durvalumab (MED14736), a PD-L1 antibody, is being evaluated in 3 phase III studies: (1) alone in patients with completely resected NSCLC, (2) alone in patients with locally advanced unresectable (stage III) NSCLC who have not experienced progression after chemoradiation therapy (PACIFIC), and (3) alone or with tremelimumab, a CTL-4 checkpoint inhibitor, versus standard of care therapy in patients with locally advanced or metastatic NSCLC who had received prior therapy and have no known EGFR and ALK mutations (ARTIC).

Several additional phase III studies are evaluating the 2 CTL-4 checkpoint inhibitors ipilimumab and tremelimumab:

- A phase III study of nivolumab versus nivolumab plus ipilimumab versus chemotherapy in patients with stage IV NSCLC (CheckMate 227)

- A phase III study of durvalumab with and without tremelimumab versus standard-of-care chemotherapy for patients with NSCLC (Mystic)

- A phase III trial of durvalumab plus tremelimumab versus standard-of-care chemotherapy for patients with NSCLC (NEPTUNE)

Other immunotherapies include the development of (1) monoclonal antibodies, (2) therapeutic vaccines, and (3) adoptive cell therapy.

Because serious immune-related adverse events are different from the toxicities associated with the administration of chemotherapy, physicians, nurses, and other health care providers must understand what the toxicities are and how to treat them.

These toxicities include issues in numerous systems. Adverse events in the endocrine system include thyroiditis, hypothyroidism, hyperthyroidism, hypophysitis, hypopituitarism, and adrenal insufficiency. In the pulmonary system, they can include pneumonitis and respiratory failure. In the gastrointestinal system, they include nausea, emesis, diarrhea, colitis, perforation, and pancreatitis. Neurologic adverse events include neuropathy, meningitis, and Guillain-Barré syndrome; ocular events include iritis, uveitis, and conjunctivitis; cardiac events include pericarditis; dermatologic events include mucositis, rash, and vitiligo; hepatic concerns include transaminitis; and renal toxicities include nephritis and renal insufficiency. ${ }^{10}$

Significant progress has been made with the use of immunotherapy in the treatment of lung cancer. Much work is still needed to develop optimal doses and combinations for different stages of lung cancer. A reliable predictive biomarker of the therapy's effectiveness needs to be established. Is the PD-1 expression according to proportion score reliable? The various pharmaceutical companies that have one 
of these checkpoint inhibitors and abnormalities of PD-L1 expression should work together to achieve this goal.

The cost of immunotherapy is high and the issue of cost-effectiveness must be addressed by all the parties affected-patients, health insurers, pharmaceutical companies, and government. Stay tuned. The future is now! It is important that we succeed for the improved health of patients with lung cancer.

\section{References}

1. Ettinger DS, Cox JD, Ginsberg RJ, et al. NCCN Clinical Practice Guidelines in Oncology for Non-Small Cell Lung Cancer. Oncology 1996;10(Suppl 11):81-111.

2. Siegel RL, Miller KD, Jemal A. Cancer statistics. CA Cancer J Clin 2016;66:7-30.

3. Ferlay J, Soerjomataram I, Ervik M, et al. GLOBOCAN 2012 v1.0, Cancer Incidence and Mortality Worldwide: IARC CancerBase No. 11 [Internet]. Lyon, France: International Agency for Research on Cancer; 2013. Available at: http://globocan.iarc.fr. Accessed July 20, 2016.

4. Ribas A. Releasing the brakes on cancer immunotherapy. N Engl J Med 2015;373:1490-1492.

5. Brahmer J, Reckamp KL, Baas $P$, et al. Nivolumab versus docetaxel in advanced squamous-cell non-small-cell lung cancer. N Engl J Med 2015;373:123-135.

6. Gettinger SN, Horn L, Gandhi L, et al. Overall survival and long-term study of nivolumab (anti-programmed death 1 antibody, BMS 936558, ONO-4538) in patients with previously treated advanced non-small-cell lung cancer. J Clin Oncol 2015;33:2004-2012.

7. Borghaei H, Paz-Ares L, Horn L, et al. Nivolumab versus docetaxel in advanced nonsquamous non-small-cell lung cancer. N Engl J Med 2015;373:1627-1639.

8. Garon EB, Rizvi NA, Hui R, et al. Pembrolizumab for the treatment of non-small-cell lung cancer. N Engl J Med 2015;372:2018-2028.

9. Herbst RS, Baas P, Kim DW, et al. Pembrolizumab versus docetaxel for previously treated, PD-L1-positive, advanced non-small-cell lung cancer (KEYNOTE-010): a randomised controlled trial. Lancet 2016;387:1540-1550.

10. Postow M, Wolchok J. Toxicities associated with checkpoint inhibitor immunotherapy. Available at: http://www. uptodate.com/contents/toxicities-associated-with-checkpoint-inhibitor-immunotherapy. Accessed July 20, 2016. 